\title{
Building energy information systems: user case studies
}

\author{
Jessica Granderson • Mary Ann Piette • \\ Girish Ghatikar
}

Received: 18 February 2010 / Accepted: 17 May 2010/Published online: 16 June 2010

(C) The Author(s) 2010. This article is published with open access at Springerlink.com

\begin{abstract}
Measured energy performance data are essential to national efforts to improve building efficiency, as evidenced in recent benchmarking mandates, and in a growing body of work that indicates the value of permanent monitoring and energy information feedback. This paper presents case studies of energy information systems (EIS) at four enterprises and university campuses, focusing on the attained energy savings, and successes and challenges in technology use and integration. EIS are broadly defined as performance monitoring software, data acquisition hardware, and communication systems to store, analyze, and display building energy information. Case investigations showed that the most common energy savings and instances of waste concerned scheduling errors, measurement and verification, and inefficient operations. Data quality is critical to effective EIS use, and is most challenging at the subsystem or component level, and with nonelectric energy sources. Sophisticated prediction algorithms may not be well understood but can be applied quite effectively, and sites with custom benchmark models or metrics are more likely to perform analyses external to the EIS. Finally, resources and staffing
\end{abstract}

J. Granderson $(\bowtie) \cdot$ M. A. Piette $\cdot$ G. Ghatikar

Lawrence Berkeley, National Laboratory,

1 Cyclotron Road,

Berkeley, CA 94720, USA

e-mail: JGranderson@lbl.gov were identified as a universal challenge, indicating a need to identify additional models of EIS use that extend beyond exclusive in-house use, to analysis services.

Keywords Anomaly detection · Baselining · Benchmarking · Energy efficiency · Diagnostics · Energy analysis · Energy information system · Enterprise energy management · Performance monitoring $\cdot$ Web-based energy management and control system

\section{Introduction}

The focus of this research is case studies of the use of energy information systems (EIS) in commercial enterprises and university campuses. Specifically, it aims to understand use of the technology to improve energy performance in buildings. EIS are broadly defined as performance monitoring software, data acquisition hardware, and communication systems used to store, analyze, and display building energy data. Time-series data from meters, sensors, and external data streams are used to perform analyses such as baselining, load profiling, benchmarking, and building level anomaly detection. At a minimum, EIS provide hourly whole-building electric data that is web accessible, with analytical and graphical capabilities (Motegi and Piette 2003), as illustrated in Fig. 1. 


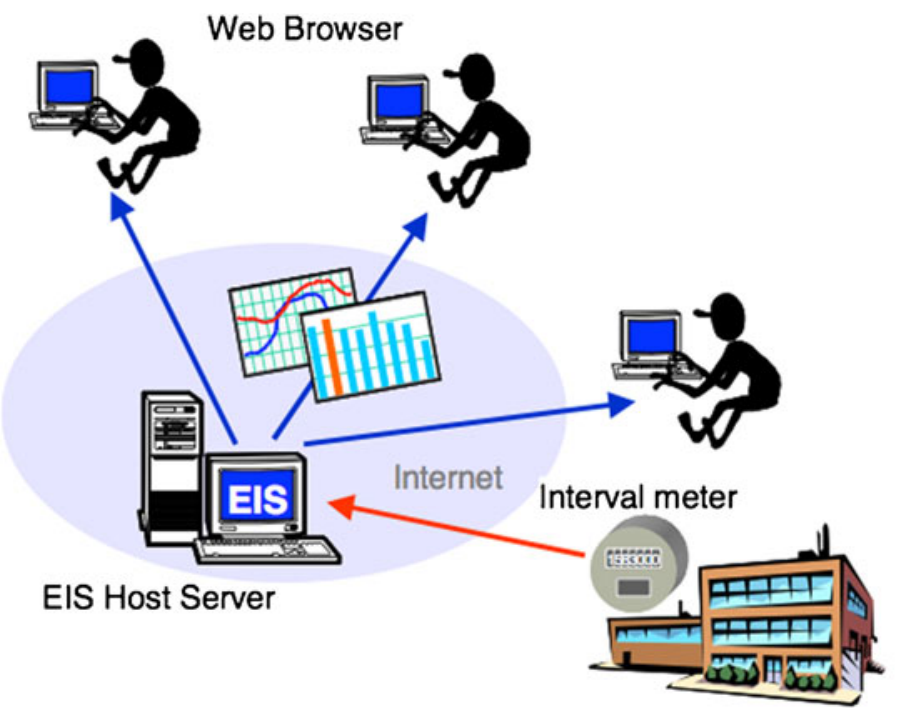

\section{Basic features}

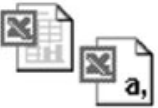

Data Acquisition

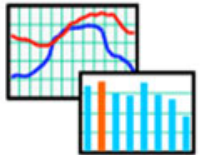

Graphical Visualization

Fig. 1 Basic Energy Information System (Motegi and Piette 2003)

EIS may also include submeter, subsystem, or component level data, and corresponding analyses such as system efficiencies or analysis of end use, yet these are less common.

EIS are viewed as a promising technology to reduce energy use in buildings, for a number of reasons. There is widespread recognition that there is often a large gap between building energy performance as designed, and measured post-occupancy energy consumption (Diamond et al. 2006; Johnson 2002; New Buildings Institute 2008). A growing body of evidence points to the value of permanent metering and monitoring (Piette et al. 2001b), particularly in the context of monitoring-based and continuous or retro-commissioning (Brown et al. 2006; Mills et al. 2005; Mills and Mathew 2009). Also pointing to the value of monitoring, researchers have increasingly documented the positive behavioral impacts of making energy consumption visible to building occupants and residents (Darby 2006; Petersen et al. 2007). In addition, EIS are well aligned with current trends toward benchmarking and performance reporting requirements.

\section{Research questions and prior work}

The case studies that are presented reveal critical aspects of EIS usability, and the interplay between features, diagnostics, and energy saving actions.
Research questions motivating this work include: Who uses EIS, and which features have proved most useful in attaining energy savings? What actions are taken based on the information provided via an EIS? How much of a building's low energy use or energy savings can be attributed to the use of an EIS? What are common challenges encountered in whole building performance monitoring? What are successful, realistic EIS implementation and use models?

The body of prior work dedicated to EIS is sparse compared to other aspects of building control and diagnostics, yet there are several studies that merit attention. A recent study (Granderson et al. 2009) assessed the state of the technology, updating older work (Motegi and Piette 2003) to identify the full spectrum of analyses and diagnostics that EIS support, and standard features versus more sophisticated functionality. Two books published in 2005 and 2007 contain editors' compilations of articles that document the implementation of web-based building control and automation systems and their use for enterprise or site energy analysis (Capehart and Capehart 2005; Capehart et al. 2007). Last year, New Buildings Institute published a report that considers EIS in the context of advanced metering technologies (New Buildings Institute 2009). The California Energy Commission has published EIS costs and qualitative benefits in the context of enhanced automation (California Energy Commission 
2002), and Lawrence Berkeley National Laboratory has studied EIS and system-specific performance monitoring and diagnostics (Motegi and Piette 2003; Motegi et al. 2003; Piette et al. 2001a, b). Finally, a substantial body of work is dedicated to facilities' and service providers' use of building automation systems, or energy management and control systems. However, it tends to focus on questions concerning the use of HVAC data for applications external to the EMCS, and on HVAC performance diagnostics and fault detection (Friedman and Piette 2001; Heinemeier 1994; Webster 2005).

Case studies documenting EIS use are modest and commonly comprise vendor-authored publications that are written to publicize successful implementation of a specific EIS, and to emphasize positive aspects of the technology that the vendor wishes to advertise. Case studies from the building energy analysis community are more objective in their assessments, and more varied in the content and level of detail. Integration and installation, and the use of EMCS data are more frequently asked about (Capehart and Capehart 2005; Capehart et al. 2007; Webster 2005) than the relationship between software features, actions taken, and resulting energy savings (Motegi et al. 2003). When features actions and savings are addressed in the literature, the overall topic is usually not whole-building EIS diagnostics, but rather equipment and system level operational diagnostics.

This paper begins with a description of the methodology used to conduct the case studies, followed by specific findings for each of the four cases. The final two sections of the paper are dedicated to research conclusions and directions for future work. Case study sites were identified through local contacts and interviews with EIS vendors, and all reported findings are based on information supplied by the case study organizations at the time that the research was conducted. Moreover, as the intent of this research is to evaluate the use of EIS technology, inclusion in the paper does not imply endorsement of the EIS or of the participating organizations.

\section{Case study methodology}

Wal-Mart Stores, Inc., Sysco Corporation, the University of California, Berkeley, the University of
California, Merced were selected for case study based on the following criteria: users with a high level of engagement with energy data and a role in energy management, aggressive savings or high-efficiency performance, and willingness to participate in 3-4 h of interviews and site visits, location permitting. Although each case was unique, a common set of information was sought from each, including the purpose and frequency of typical EIS use; how EIS information is shared throughout the organization, or across different users; challenges in implementing or learning the technology; and quantified energy savings or operational changes that resulted from use of the technology. The UC Merced case study was conducted with the campus energy manager, and the UC Berkeley case included the associate director of sustainability and engineering services, and members of her staff groups. The Sysco case study was informed by the energy services provider and the employee who is accountable for energy performance at a Northern California warehouse site. The WalMart case combined discussions with a benchmarking analyst from the Energy Department, the Electrical Engineering Manager from Prototypical Design/ Construction Standards, and the Senior Manger of Energy Systems and Technology Development.

As summarized in Table 1, the four cases that were chosen represent commercial enterprises and university campuses with a diversity of performance monitoring technologies, commercial building types, and portfolio sizes. These cases encompass buildings that range from Wal-Mart and Sysco's relatively repeatable warehouse and retail designs, to UC Berkeley's legacy and historic sites, to UC Merced's very-low energy new construction (New Buildings and with the California Institute for Energy and Environment 2009). In the following, sections three through six, case-specific findings are presented.

\section{Sysco (Sygma)}

Between 2005 and 2006 Sysco Corporation, North America's largest foodservices distributor, implemented a 3-year corporate-wide energy efficiency program with a target of $25 \%$ reduction in energy consumption across a portfolio of 143 distribution centers in the US and Canada. Sysco has a longstanding energy services contract with a large 
Table 1 Case study sites and characteristics

\begin{tabular}{lllc}
\hline Case & Type $\left(\mathrm{m}^{2}\right)$ & Controls & Performance monitoring \\
\hline $\begin{array}{l}\text { Sysco, Stockton CA } \\
\text { Sygma site }\end{array}$ & $\begin{array}{c}\text { Refrigerated/dry warehouse } \\
(4.8, \text { Stockton } 8,800)\end{array}$ & DOS-based refrigeration control & $\begin{array}{c}\text { NorthWrite Energy WorkSite } \\
\text { Utility bills }\end{array}$ \\
$\begin{array}{l}\text { Wal-Mart, USA } \\
\text { Retail/grocery }(63 \mathrm{M})\end{array}$ & $\begin{array}{c}\text { Novar Danfoss Emerson Computer } \\
\text { Process Controls }\end{array}$ & $\begin{array}{c}\text { Energy ICT EIServer Utility } \\
\text { bills }\end{array}$ \\
$\begin{array}{l}\text { UC Merced, Merced } \\
\text { CA }\end{array}$ & Campus $(83,000)$ & $\begin{array}{c}\text { Automated Logic Corporation (ALC) } \\
\text { WebCTRL }\end{array}$ & ALC WebCTRL Utility bills \\
CA Berkeley, Berkeley & Campus $(1.5 \mathrm{M})$ & Barrington Some ALC, Siemens & Obvius Utility bills \\
\hline
\end{tabular}

engineering firm, with whom a three-part approach was adopted to identify and implement energy-saving changes. First, site visits by expert refrigeration engineers and technicians were conducted for tuneups and to identify low/no cost energy-saving measures. Second, the EIS was customized to accommodate Sysco's goals; and third, continuous communication and collaboration was supported between corporate managers, the engineering firm, and the on-site 'energy champions' at each warehouse. This approach has enabled Sysco to outpace its goal, reaching $28 \%$ reductions before the end of the program period $(10 \%$ from capital improvements and upgrades), with monthly savings of roughly $18,000,000 \mathrm{kWh}$. While the UC Merced case revolved around the particular constraints and power of a densely populated, sophisticated web-EMCS platform, the Sysco case highlights: (1) classic enterprise-wide EIS use and information sharing, (2) limited yet powerful on-site use of the EIS, (3) use of EIS technology to ensure persistence in savings and energy accountability.

\section{Sysco EIS uses and challenges}

Sysco uses NorthWrite's Energy WorkSite EIS. Pulse outputs from monitored electric utility meters are communicated via cellular to a central database. Sysco performs both site-specific and portfolio analyses on a monthly basis, using the EIS embedded reporting capabilities. The engineering firm inputs utility billing invoices into the database and portfolio benchmark rankings are generated as listed in Table 2. Managers coordinate monthly group reviews with each site's 'energy champion', who is accountable for energy use. Monthly rankings are compared based on a metric called the efficiency factor, which takes into account wet bulb temperature, the total volume of frozen and refrigerated space, total and daily energy consumption, and weather predicted energy performance. Although not deeply understood by energy champions and managers, the efficiency factor is the basis of site benchmarking. It is a custom defined metric developed for Sysco's portfolio of refrigerated warehouses, and preconfigured within the EIS reporting tools. Sysco also tracks $\mathrm{kWh} / \mathrm{sf}$ relative to the start of the efficiency program.

The Sysco site visit was conducted at the Stockton, CA Sygma distribution center. Stockton ranks highly in the Sysco portfolio, and has reduced site energy use by $36 \%$ since the start of the efficiency program in 2005. The energy champion makes near-exclusive use of the 'meter monitor' to manage his most energy intensive space. As shown in Fig. 3, this view contains a two-point overlay comparing the current week's or day's time series to that of the prior week, and summary of relative consumption and ambient temperatures. The Stockton energy champion uses this information display to implement a daily energy efficiency strategy. The existing controls do not permit it; however, the frozen goods can tolerate fluctuations in temperature between -5 and $10^{\circ} \mathrm{F}$ for short periods of time, without compromising quality. In response, refrigeration setpoints are manually raised each morning to force the compressors to shut down for several hours, thereby saving energy. The lighter-colored trend in Fig. 2 reflects an instance of this daily strategy, whereas the darker line reflects a day in which the energy champion was on vacation and the strategy was not implemented. In this instance, in spite of a temperature increase, the average load was reduced by approximately $35 \%$ throughout the morning, relative to a day in which strategy was not implemented. 
Table 2 Sysco metrics, benchmarks, and data sources

\begin{tabular}{llll}
\hline EIS & Performance metrics & Data sources & Benchmarks \\
\hline NorthWrite & Unit-less efficiency factor & Electric utility meter & Portfolio rankings based on efficiency factor \\
Energy WorkSite & Annual, monthly kWh/ksf & Utility invoices & Pre-program consumption [kWh/sf] \\
& & Weather feed & \\
\hline
\end{tabular}

Though the meter dedicated to refrigeration loads is the primary focus of EIS use, minor energy management tasks are performed with all meters. Unanticipated and unexplained spikes in consumption are identified and reviewed with equipment technicians, and deviations from expected profiles are investigated and addressed. Sysco monitors electric utility meters and has not pursued submetering, with the result that aside from infrequent minor glitches in cellular communications, data quality has not been a critical challenge.

The Stockton site visit revealed that much of the EIS functionality was unused and unexplored. The highly customized implementation of the EIS configured to meet Sysco's needs, and the collaboration with expert engineering service providers has resulted in a successful efficiency program based on straightforward information, and frequent energy-based communication throughout the organization. Successful measures implemented during the initial stages of the program, accountability based on monthly reporting, and an emergent corporate culture of competition, have provided significant value. At the Stockton site the EIS is most highly valued for its role in supporting and encouraging accountability and motivating staff so that efficiency gains might persist over time. However it is possible that additional energy savings have gone unidentified because energy champions have not seen the value in the full set of EIS capabilities.

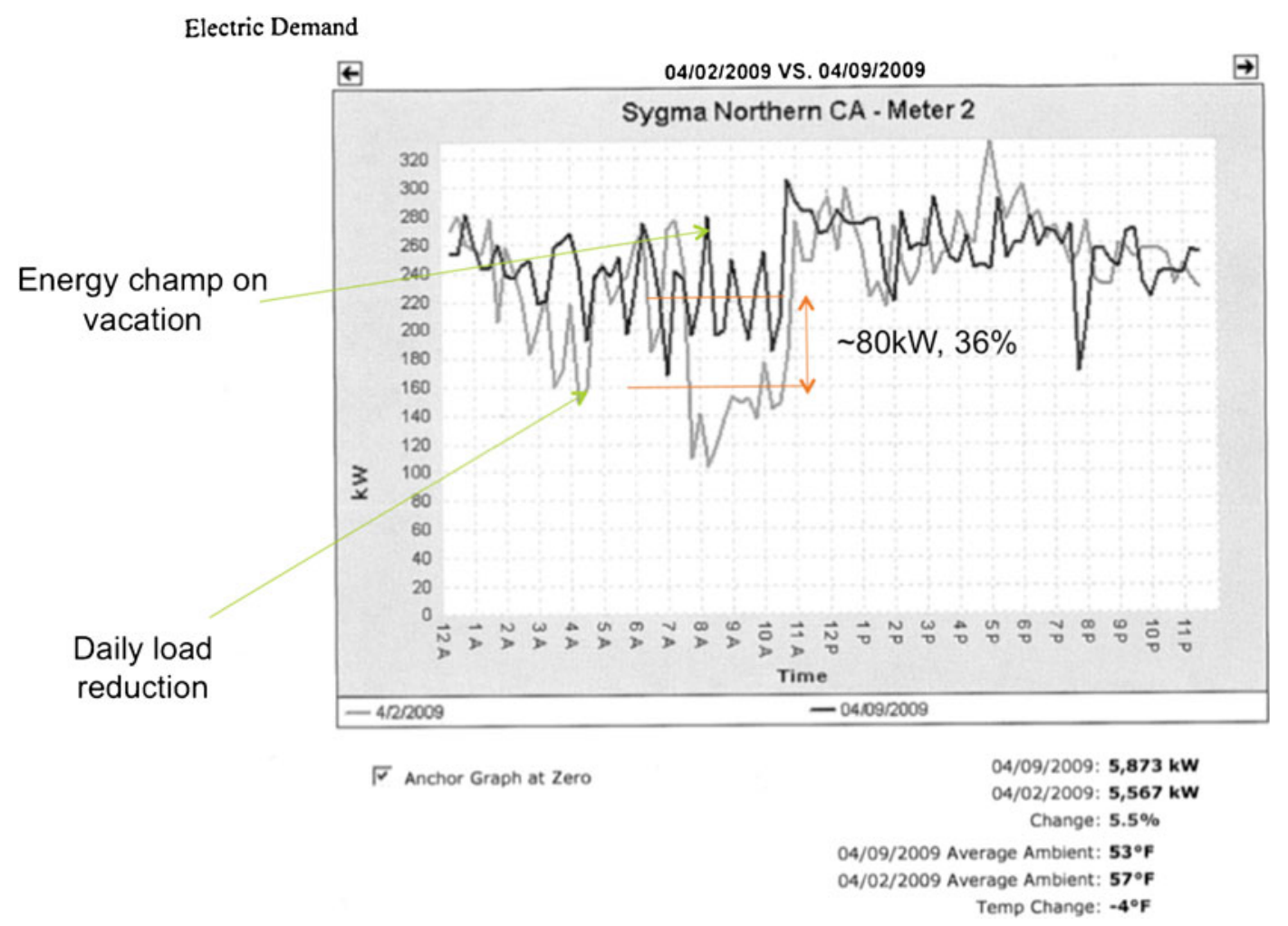

Fig. 2 Energy WorkSite's 'meter monitor' and Stockton Sygma efficiency strategy 


\section{Wal-Mart}

Wal-Mart maintains a portfolio of 67 million $\mathrm{ft}^{2}$ of commercial retail space in the US, and uses Energy ICT's EIServer to collect and monitor energy consumption data. Wal-Mart's decision to implement an EIS was motivated by an overarching business philosophy that holds that with billion dollar utility expenses, energy information limited to 60 - or 90-day billing cycles is insufficient to manage energy use. Wal-Mart's Energy Systems and Technology Development manager and building design engineers analogize that they would never base retail decisions on 60-day-old sales data, and that energy considerations are just as critical.

The central themes highlighted in the Wal-Mart case contrast markedly to those at Sysco. Rather than integrated EIS use throughout the enterprise to meet portfolio goals, as at Sysco, Wal-Mart is a case of EIS use by specific groups or individuals for a few key purposes, among various departments and teams in the enterprise. Benchmarking, measurement and verification $(\mathrm{M} \& \mathrm{~V})$, and power procurement and demand response are the most common uses. A group of internal supporters champion the use of the EIS technology and maintain a vision for how its use might be expanded throughout the organization; regular operational analytics are not yet widespread vertically or horizontally within the enterprise. In addition, Wal-Mart illustrates the fact that even the more sophisticated EIS may not satisfy all of an organization's analytical and energy performance monitoring needs. For uses such as M\&V EIServer's embedded functionality is well suited to user needs, while for others such as portfolio benchmarking, the EIS data is exported to third party software for analysis.

\section{Wal-Mart EIS uses and challenges}

Wal-Mart's EIS data come from independent meters that 'stand alone' from the building management systems. HVAC, lighting, and refrigeration electric mains are typically metered, and some stores monitor gas and water as well. Data from a subscription weather feed is imported into the EIS on a real-time basis. Store and portfolio performance metrics are summarized in Table 3.

EIServer features a custom module for M\&V tasks that has been used extensively at Wal-Mart, although on an ad hoc basis, to determine the effectiveness of energy efficiency improvements. Regression analyses establish weather-normalized baseline forecasts against which actual measured consumption data are compared. The wholesale power procurement and demand response group makes considerable use of EIServer's forecasting and normalization features, with experience indicating that the technology is sufficiently accurate for week-ahead predictions, and accurate to within to within $1 \%$ for hourly time intervals.

At the individual store level, the EIS is used to gauge the performance of new designs, particularly at 'High Efficiency' supercenters that target 20-45\% savings versus the typical Wal-Mart store ${ }^{1}$. However, one user does report that High Efficiency stores are best analyzed by exporting EIS data for use in Integrated Environmental Solutions Virtual Environment models, because of the ability to run computational fluid dynamics, solar thermal, and daylighting simulation modules. Wal-Mart applies a custom model-based approach to calculate weather and sales normalized energy use intensities, therefore EIS data are exported to external software for portfolio energy tracking. Each month, the benchmarking analyst identifies the 20 poorest performing sites, and refers them for further investigation at the operations and maintenance level. In some cases, the benchmarking analyst explores the data for an individual store. However, she does not rely upon the EIS normalization capability, preferring to control comparisons by selecting stores from similar climates.

$\mathrm{M} \& \mathrm{~V}$ and benchmarking activities provide two examples of energy savings attributable to Wal-Mart's use of the EIS. At one site, poor store performance was traced back to a $225 \mathrm{~kW}$ static lighting load attributable to a failed dimming control module. Figure 3 shows the lighting submeter trend used to identify this failure. As an example of M\&V uses, the EIS Project Tracker module has been used to identify several sites in which a failed or incorrectly installed VFD prevented actual energy savings.

Wal-Mart's EIS challenges are largely independent of the EIS technology itself. Submetering has been difficult because it has not been financially feasible to meter each store to the degree desired by the corporation's internal EIS champions. Given that the average supercenter contains a dozen submeters,

\footnotetext{
${ }^{1}$ Wal-Mart Stores, Inc.-Sustainable Buildings. http://walmart stores.com/Sustainability/9124.aspx. Accessed January 2010.
} 
Table 3 Wal-Mart metrics, benchmarks, and data sources

\begin{tabular}{llll}
\hline EIS & Performance metrics & Data sources & Benchmarks \\
\hline Energy ICT & Weather and sales normalized $\mathrm{kWh} / \mathrm{sf}$ & Building and submetered electric & Portfolio rankings \\
EIServer & Retrofit energy savings & Some gas and water & Pre-retrofit baseline \\
& DR power and cost savings & Subscription weather feed & DR load shapes and baselines \\
& & ICT project tracking & \\
\hline
\end{tabular}

consistency in the quality of contracted installations has also been a concern. More central to understanding real-world EIS use, Wal-Mart has faced difficulty integrating regular EIS use into standard daily activities, particularly during the current economic downturn. For example, supporters of the EIS technology would like to see at a minimum, that all staff has access to the system through web-based executive reporting. Similarly, one person currently performs benchmarking tasks every 30 days, whereas the vision is to support a benchmarking group that would engage with the data on a daily basis.

\section{UC Merced}

Having opened in 2005, UC Merced (UCM) is the newest University of California campus. Prior to opening, the campus made a strong commitment to energy-efficient building design, and energy conservation plays a fundamental role in campus objectives (New Buildings and with the California Institute for Energy and Environment 2009). UC Merced features a uniquely dense metering and monitoring infrastructure, based on the campus web-EMCS. A web-EMCS is defined as an Internet-accessible system used to operate building systems (usually HVAC) through a series of sensors and controllers (Motegi and Piette 2003). Over 10,000 control and energy monitoring points are currently trended at UCM's three academic buildings, the central plant, and smaller auxiliary buildings. Custom benchmarks and deep monitoring capability with a sophisticated web-EMCS are central to the main themes embodied at the campus: (1) the use of a web-EMCS as an EIS and (2) the web-EMCS as enabling critical information links and realization of the campus as a living laboratory for energy efficiency analyses.

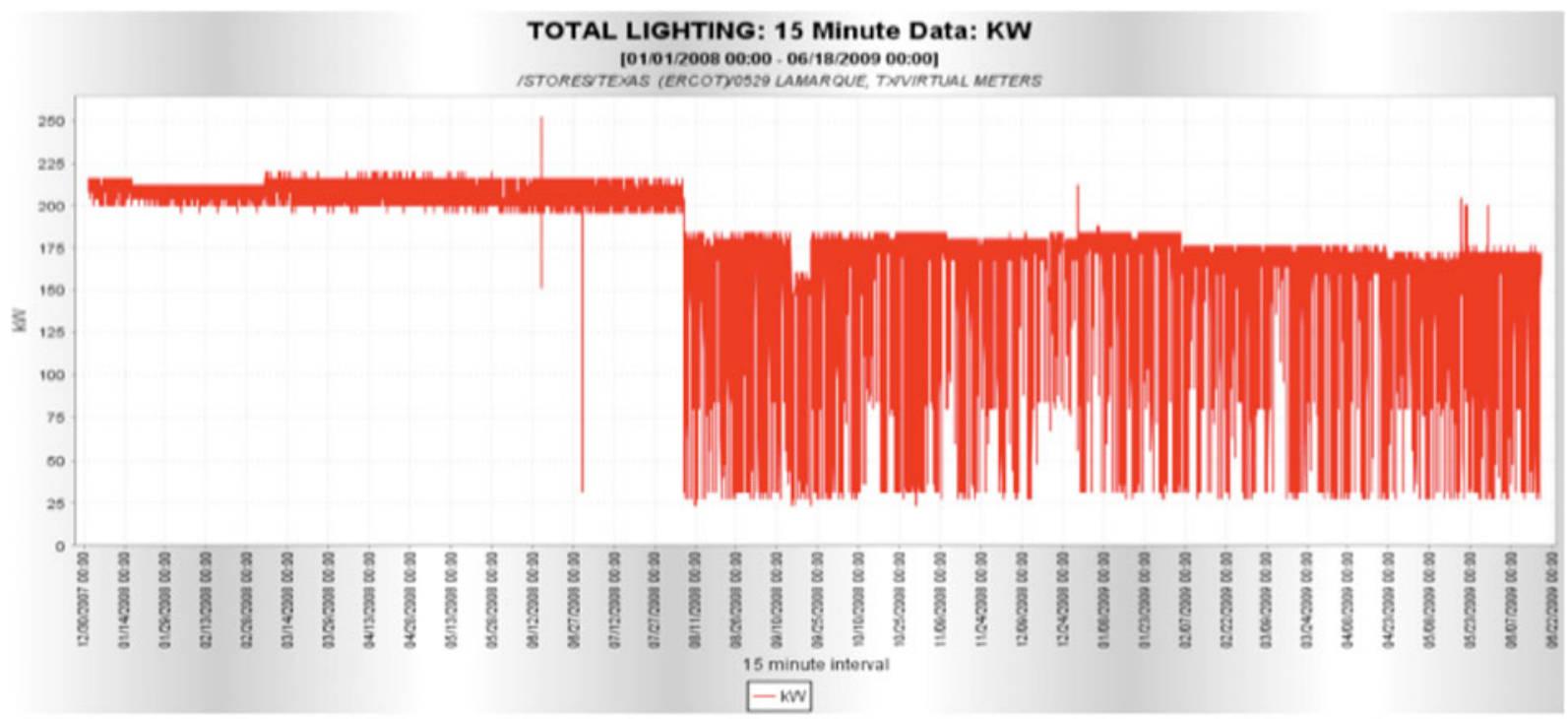

13 Sub-Metering

Fig. 3 Non-functional dimming module at Wal-Mart identified with EIServer, and then fixed 


\section{UC Merced web-EMCS uses}

Typically, use of the WebCTRL web-EMCS at UC Merced is dominated by operational investigations. Use of the web-EMCS for traditional EIS analyses directed at campus and whole-building energy performance has been complicated by the fact that the EMCS and monitoring instrumentation were not explicitly commissioned or configured to track key energy performance metrics. The metrics used to track energy performance are more complicated than simple energy use intensities (Brown 2002), and the logic-based arithmetic in WebCTRL was not initially programmed to perform the associated calculations. WebCTRL meter data are used to track annual energy performance; 15-min gas, electricity, hot water, and chilled water consumption are measured at the campus level and for critical buildings, as summarized in Table 4. On a monthly basis, the campus energy manager uses the web-EMCS data to determine utility recharges for non-state buildings (that is, buildings that are differently financed, requiring that they "reimburse" the campus for utilities).

Lessons from analysis of the campus steam system provide an example of how the web-EMCS was used to identify operational changes leading to energy savings. Gas trends at the central steam plant showed significant gas use throughout the night when the system was not intended to operate. At the same time, steam trends at the central plant revealed non-zero operating pressures at night. The energy manager shared the data with the superintendent, who subsequently modified the system to true zero overnight pressure securing a $30 \%$ reduction in average daily gas consumption at the steam plant, and an estimated
$\$ 2,500$ monthly savings (308 MBtu/month). In the lower portion of Fig. 4, the change to zero overnight pressure is plotted; in the upper portion, the resulting drop in overnight gas use is shown.

In addition to providing a rich set of operational and energy consumption data the web-EMCS has also facilitated the use of the campus as a living laboratory. To this end the web-EMCS data are used in several ways. For example the data are used in thermodynamics course modules that the energy manager teaches. The data are also used to inform student and faculty research efforts, and in short and long-term research and demonstration collaborations with the external buildings research community.

UC Merced challenges and needs

Data quality issues arise in a number of contexts at UC Merced. Networking and connectivity problems have led to mis-communicated values that generate errors, lock out equipment, and cause large volumes of false data and cascading false alarms. While not attributable to the capabilities of the EMCS, metering and sensor calibration and configuration errors greatly impact data quality, thereby impacting the ability to use the EMCS as an EIS. With the exception of whole building electric data, significant resources were required to manually validate the EMCS data and to quantify energy performance relative to benchmarks.

At UC Merced, the energy manager has not been able to investigate whole-building and submetering end-use trends to the full extent desired; and campus wide, it has taken some effort to transition from reactive to proactive use of the data. Note that prior research in the use of building management systems

Table 4 UC Merced metrics, benchmarks, and data sources

\begin{tabular}{llll}
\hline Web-EMCS & Annual building metrics & Data sources & Benchmarks \\
\hline Automated & Peak electric demand & Building electric meter & $\begin{array}{l}\text { UC/CSU weather and } \\
\text { building-type normalized } \\
\text { energy use intensity } \\
\text { (Brown 2002) }\end{array}$ \\
Logic & Total electric use & & Central plant electric submeters \\
Corporation & Peak chilled water demand & Central plant gas meters & Building gas meters \\
WebCTRL & Total gas use (incl. steam and HW) & Building chilled/hot water flow, \\
& & and supply/return temperature & \\
& & Utility bilss & \\
\end{tabular}



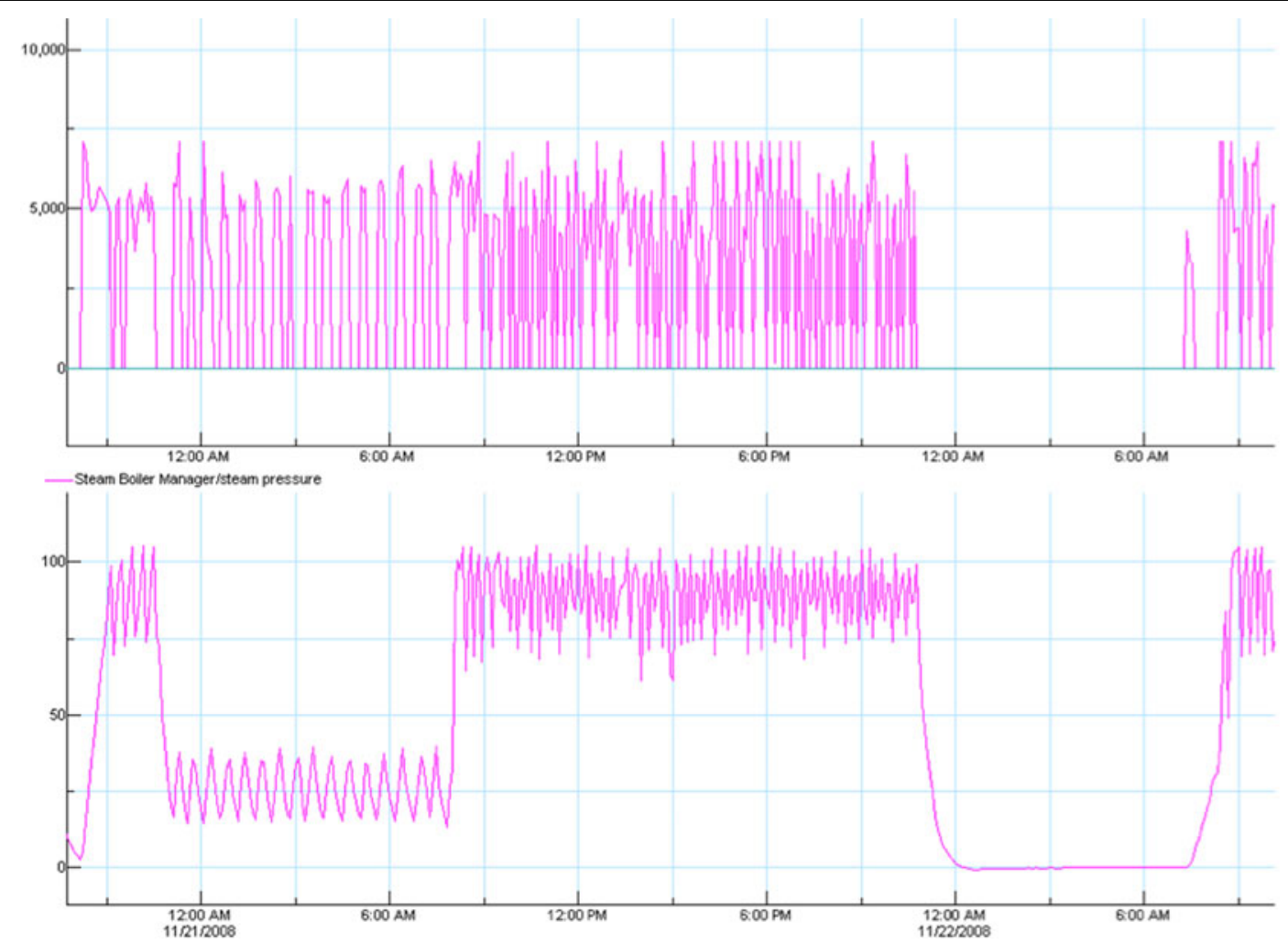

Fig. 4 Web-EMCS trends and energy saving operational change at UC Merced

at government buildings identified similar challenges in proactivity, resources, and energy management (Webster 2005). Overall, the energy manager reports a high level of satisfaction with WebCTRL emphasizing that UC Merced trends extremely large volumes of data and that intensive monitoring needs to be undertaken deliberately, with close attention to a spectrum of issues including wiring, system programming, network architecture, and hardware selection.

\section{UC Berkeley}

The University of California, Berkeley (UCB) is a 140-year-old, 15.9 million $\mathrm{ft}^{2}$ campus with a wide diversity of building ages, types, and sizes. Campus energy performance has been prioritized to differing degrees throughout the last decade, and Berkeley is currently experiencing a period of renewed attention to efficiency. There is no central EIS at UCB. UCB is a contrasting case relative to the other three that is included to illustrate the challenges that are encountered in the absence of campus-wide performance monitoring. It also provides insights as to the information needs and energy management desires of a specific energy manager, when a large ageing campus is tasked with reducing its climate impact. Although there is no campus EIS, there is a large volume of energy and system performance data.

UC Berkeley data uses and needs

The utility group uses utility bills and monthly manual meter reads to manage the purchase and billing of all campus energy. They process all invoices, and perform accounting reviews for approximately 200 utility accounts, including water, electric, gas, and steam. The EMCS group at UC Berkeley uses Broadwin's WebAccess Project Manager to remotely oversee the campus control systems. Each day eight person hours are dedicated to building-bybuilding HVAC equipment checks across 61 buildings. Appropriate on/off status and setpoints are verified, and when problems are detected, the staff delves further into time-series plots of relevant trend data. 
A number of campus efficiency and commissioning interventions have implemented remotely accessible electric interval metering at approximately 20 buildings. A 15-min interval data can be visualized, plotted, or exported using a web application. A potentially useful tool is under development in a student-funded research project. The Building Energy Dashboard includes monthly representations of energy, water, and steam, and real-time displays of the remote access building electric meters. Figure 5 shows the 'detailed building plot' in which this week's consumption is plotted against the previous week, with minimum maximum and average demand $^{2}$. Student trials using such plots revealed excessive operation of the ventilation system and over illumination in the architecture building. Based on these observations, the ventilation schedule was reduced by $6 \mathrm{~h}$ per day, and a lighting de-lamping was conducted, resulting in a $30 \%$ reduction in total energy use. The two trends in Fig. 5 show the whole-building power before and after these changes were made. As the dashboard combines data from the utilities group with interval data that is currently used only on a limited basis, the application might be useful for the campus energy management team as well as for building occupants.

Similar to the other cases in the study, resources were cited as a challenge at UC Berkeley. In particular, the energy manager prioritizes tracking performance at the building level, and providing feedback to building coordinators, EMCS and HVAC staff, and technicians. She also would like more remote-access interval metering, submetering beyond the whole-building level, and access-controlled public data.

\section{Discussion}

The case studies that were presented attempted to answer questions related to energy savings and actions attributable to EIS use, performance monitoring challenges, and successful implementation models. As the associated conclusions overlap considerably, they are grouped into energy savings, organizational impacts and success factors, and usability and analysis. Table 5 summarizes actions that were taken based on building

\footnotetext{
${ }^{2}$ Berkeley dashboard ${ }_{\mathrm{TM}}$; analyze the campus metabolism. http://dashboard.berkeley.edu/building/ Accessed January 2010.
}

energy data in each of the cases studied, and where available, the associated energy impacts. The most common actions and observations that were encountered concerned incorrect implementation of scheduled loads, $\mathrm{M} \& \mathrm{~V}$, and inefficient or excessive operations.

Organizational impacts and success factors

The existence of data or performance monitoring software does not guarantee shared knowledge or actionable information. Enterprise-wide EIS use at Sysco has encouraged persistent savings, and a corporate culture of energy accountability, awareness, and competition. Similarly, extensive use and sharing of energy data at UC Merced has contributed to highly efficient operations and energy performance, and has supported the realization of the living laboratory concept. On the other hand, Wal-Mart and UC Berkeley are working toward more extensive use of data to reduce energy consumption.

Resources and staffing were a significant constraint in every case studied, and clearly impact the extent to which energy data is successfully used to identify energy saving opportunities. A common view is that EIS are primarily the domain of in-house staff and that services are used to a minimal degree services during installation and configuration. At the alternate end of the spectrum, EIS may be primarily intended for use by third-party energy consultants and service providers. However, the general prevalence of staffing constraints, Sysco's successful efficiency gains, and the number of EIS vendors that offer analytical services, indicate the potential for alternate models of successful EIS use.

\section{Usability and analysis}

Reliable high quality data are a critical aspect of automated analysis of building energy performance, and can have a significant impact on EIS usability. At UC Merced, failure to commission the instrumentation and web-EMCS for EIS analytics has impacted the ability to track and diagnose building performance. More generally, usability at UC Merced is affected by a number of challenges specific to implementation of an intensive monitoring infrastructure and the acquisition and storage of extreme volumes of trend data. The Merced case shows that particular attention must be paid to wiring and 


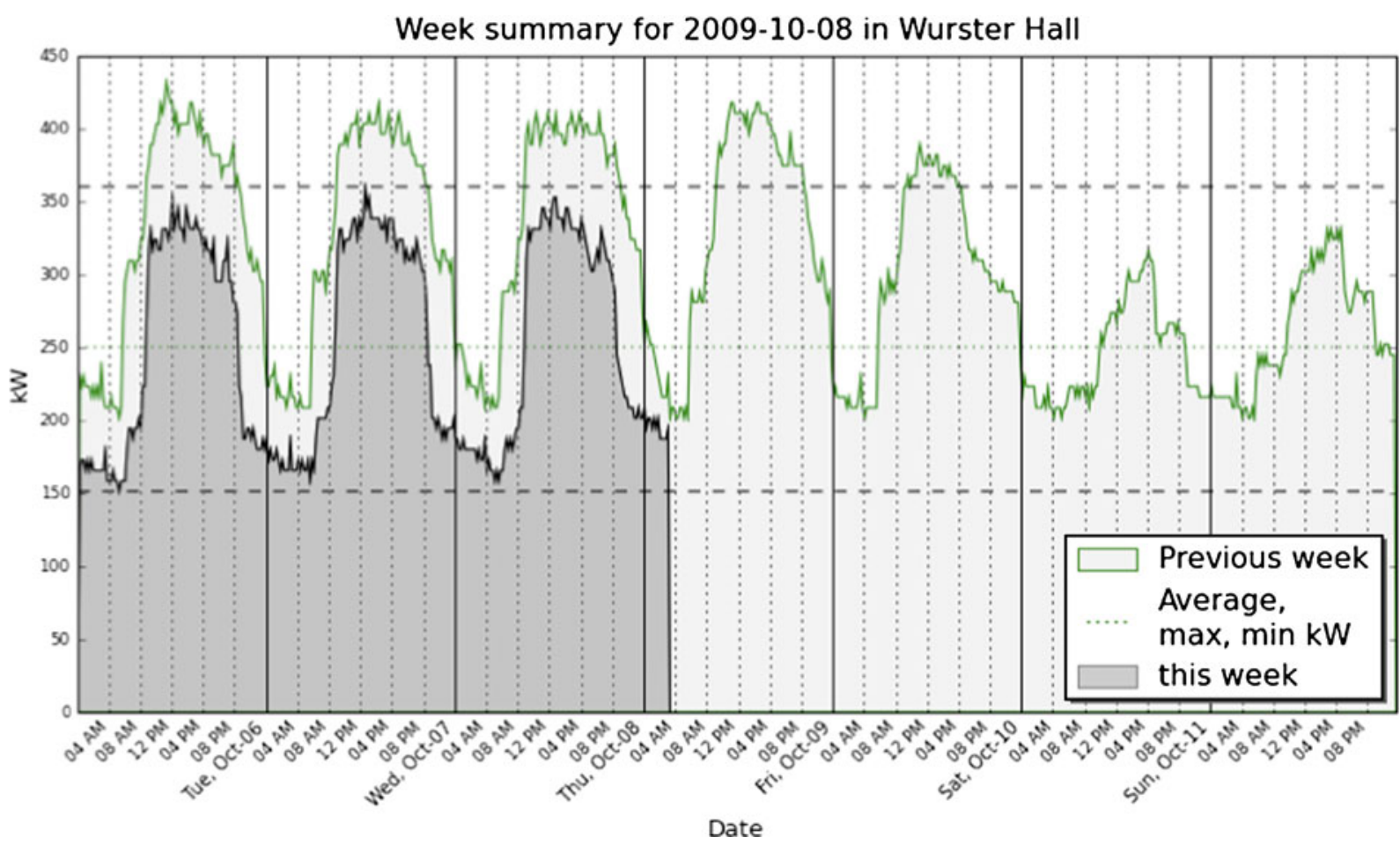

Fig. 5 UC Berkeley Building Energy Dashboard, 'detailed building plot'

Table 5 Summary of actions taken based on building energy information

\begin{tabular}{|c|c|c|c|}
\hline Site & Observation/action & EIS data points & Energy impact \\
\hline UC Merced & $\begin{array}{l}\text { Excessive overnight gas use due to } \\
\text { non-zero pressure at steam boilers }\end{array}$ & Steam plant pressure, gas & $\begin{array}{l}30 \% \text { reduction in average daily gas } \\
\text { use, } \$ 2,500 / \mathrm{mo}(308 \mathrm{MBtu} / \mathrm{mo}) \\
\text { avoided }\end{array}$ \\
\hline Sysco & $\begin{array}{l}\text { Lights left on after hours at } \\
\text { Stockton Sygma }\end{array}$ & Building electricity & \\
\hline Sysco & $\begin{array}{l}\text { Multi-hour daily energy efficiency } \\
\text { strategy at Stockton Sygma }\end{array}$ & $\begin{array}{l}\text { Building electricity, control } \\
\text { system setpoints } \\
\text { and temperatures }\end{array}$ & $\begin{array}{l}35 \% \text { demand reduction } \\
* \text { Single observation }\end{array}$ \\
\hline Sysco & $\begin{array}{l}\text { Identification of low/no cost savings } \\
\text { opportunities, e.g., retro-commissioning } \\
\text { and refrigeration tune-ups }\end{array}$ & Warehouse electric meters & $\begin{array}{l}18 \% \text { reduction in portfolio energy use } \\
36 \% \text { reduction in Stockton site energy }\end{array}$ \\
\hline UC Berkeley & $\begin{array}{l}\text { Excessive ventilation and over illumination } \\
\text { identified, leading to lighting retrofit and } \\
\text { ventilation schedule change }\end{array}$ & $\begin{array}{l}\text { Whole-building } \\
\text { electric meter }\end{array}$ & $\begin{array}{l}30 \% \text { reduction in whole building } \\
\text { energy use }\end{array}$ \\
\hline UC Berkeley & $\begin{array}{l}\text { Multi-week chiller lockout that prevented } \\
\text { shut-down }\end{array}$ & Control system setpoints & \\
\hline Wal-Mart & $\begin{array}{l}\text { Static } 225 \mathrm{~kW} \text { load at dimming control } \\
\text { submeter }\end{array}$ & Submeter electricity & $\$ 35,000 /$ year avoided costs \\
\hline Wal-Mart & $\begin{array}{l}\text { Failed or disconnected VFDs used } \\
\text { in retrofit programs }\end{array}$ & Submeter electricity & Avoided zero savings at retrofit sites \\
\hline
\end{tabular}


hardware integration, system programming, and network communications, not all of which lies wholly in the domain of the EMCS developer. In contrast, the systems used by Wal-Mart and Sysco are explicitly designed for energy analyses, and did not report significant data quality issues, likely for two reasons: EIServer has embedded validation estimation error checking routines and data quality is usually a concern only in cases of submetering and energy sources other than electric.

The degree to which a site uses embedded analytical capabilities depends on the particular performance metrics and benchmarking data that are utilized. Our cases showed that the more tailor-made the calculations, the more likely it is that the data will be exported for analysis in third party modeling or computational software. These cases indicated that sophisticated EIS normalization and forecasting methods are not universally understood across users and technology champions. Even so, these methods are commonly used to great success, in a 'black-box' manner. Finally, although EIS offer a wide range of features, actual use of these features can be very limited, and it is not clear that users are always aware of how to use the capabilities of the technology to generate energy-saving information.

\section{Summary and future research}

These case investigations have generated useful insights as to the value of EIS, to be extended in future research. Three areas of key interest are: (1) features and energy savings, (2) technology definitions and scalability, and (3) successful use and deployment models. Questions concerning the most useful features, potentially useful but underutilized features, and energy savings attributable to EIS use merit further attention. For example, a more extensive set of typical actions and associated energy savings, and documented records of building consumption before and after EIS implementation would enable stronger conclusions on the range of expected savings from EIS use. In addition, typical EIS actions and associated features can be linked to a classification of standard EIS uses such as M\&V for retrofit support, continuous building-level anomaly detection, or GHG emissions reporting. Closely related to features and usability, there is consider- able analytical potential in linking EIS anomaly detection methods to physical models. Today's EIS algorithms rely purely on empirical historic performance data to detect abnormal energy consumption. However, they do not provide a means to identify excessive energy consumption relative to the design intent, or to realize model-predictive control strategies.

From a technology standpoint, definitions and scalability require further study. The UC Merced case illustrated some of the challenges in using an EMCS as an EIS, indicating an outstanding research question - how might EMCS data acquisition and commissioning be improved so that the technology can serve as a robust EIS? Scalability is a concern that may provide insights as to where to draw the line between EIS and related technologies. In the future, it will be necessary to understand the tradeoffs between diagnostic capabilities, trend volume and number of points monitored, and the resulting burden on the system's underlying hardware and communication networks. Also related to technology definition, standardizing the format and structure of information at the data warehouse would encourage the development of advanced anomaly detection capabilities, as could the development of features to configure exported data files into formats that can be used by modeling tools such as Energy Plus or DOE-2. Standard formatting of EIS data would also facilitate the transfer of energy information from the building to outside entities, supporting and aligning with current developments in demand side management, and the smart grid.

Finally, there remains much to learn about effective EIS deployment and use models within organizations. The Sysco case reveals a potentially powerful approach in which in-house use and expert services are combined. Additional research is needed to better understand where this approach is most useful, and to determine alternate success models that are appropriate to a diversity of organizational sizes, commercial segments, and building ownership models.

Acknowledgment Vendor participation was critical to the success of this study, and the authors wish to acknowledge their generosity and willingness to be included in this work. This work was supported by the California Energy Commission and 
the California Institute for Energy and Environment under Contract No. MUC-08-04.

Disclaimer This document was prepared as an account of work sponsored by the US Government. While this document is believed to contain correct information, neither the US Government nor any agency thereof, nor The Regents of the University of California, nor any of their employees, makes any warranty, express or implied, or assumes any legal responsibility for the accuracy, completeness, or usefulness of any information, apparatus, product, or process disclosed, or represents that its use would not infringe privately owned rights. Reference herein to any specific commercial product, process, or service by its trade name, trademark, manufacturer, or otherwise, does not necessarily constitute or imply its endorsement, recommendation, or favoring by the US Government or any agency thereof, or The Regents of the University of California. The views and opinions of authors expressed herein do not necessarily state or reflect those of the US Government or any agency thereof or The Regents of the University of California.

Open Access This article is distributed under the terms of the Creative Commons Attribution Noncommercial License which permits any noncommercial use, distribution, and reproduction in any medium, provided the original author(s) and source are credited.

\section{References}

Brown, K. (2002). Setting enhanced performance targets for a new university campus: Benchmarks vs. energy standards as a reference? American Council for an Energy-Efficient Economy Summer Study Proceedings, 4, 29-40.

Brown, K., Anderson, M., \& Harris, J. (2006). How monitoring-based commissioning contributes to energy efficiency for commercial buildings. American Council for an Energy-Efficient Economy Summer Study Proceedings, 3, 27-40.

California Energy Commission. (2002). Money \& Energy Saving Resources from the California Energy Commission. Enhanced Automation Technical Options Guidebook. California Energy Commission publication \#400-02-005F. http://www.energy.ca.gov/enhancedautomation/documents/ 400-02-005F_TECH_OPTIONS.PDF. Accessed February 2010.

Capehart, B., \& Capehart, L. (Eds.). (2005). Web based energy information and control systems: Case studies and applications. Lilburn, GA: Fairmont Press, Inc.

Capehart, B., Capehart, L., Allen, P., \& Green, D. (Eds.). (2007). Web based enterprise energy and building automation systems. Lilburn, GA: Fairmont Press, Inc.

Darby, S. (2006). The effectiveness of feedback on energy consumption: A review for DEFRA of the literature on metering, billing, and direct displays. Environmental Change Institute, University of Oxford. http://www.eci. ox.ac.uk/research/energy/downloads/smart-meteringreport.pdf. Accessed November 2009.

Diamond, R., Optiz, M., Hicks, T., Von Neida, B., \& Herrera, S. (2006). Evaluating the energy performance of the first generation of LEED-certified commercial buildings. American Council for an Energy-Efficient Economy Summer Study Proceedings, 3, 41-52.

Friedman, H., \& Piette, M. A. (2001). Comparison of emerging diagnostic tools for HVAC systems. Lawrence Berkeley National Laboratory. Paper LBNL-47698. http://repositories.cdlib.org/lbnl/LBNL-47698/. Accessed November 2009.

Granderson, J., Piette, M. A., Ghatikar, G., \& Price, P. (2009). Preliminary findings from an analysis of building Energy Information System technologies. Proceedings of the National Conference on Building Commissioning. Lawrence Berkley National Laboratory. Paper LBNL-2224E. http://eis.lbl.gov/pubs/lbnl-2224e.pdf. Accessed February 2010 .

Heinemeier, K. (1994). The use of energy management and control systems to manage the energy performance of commercial buildings. Ph.D Thesis: Department of Architecture, UC Berkeley, and Energy and Environment Division, Lawrence Berkeley National Laboratory. Paper LBL-36119.

Johnson, J. (2002). Is what they want what they get? Examining field evidence for links between design intent and as-built energy performance of commercial buildings. American Council for an Energy-Efficient Economy Summer Study Proceedings, 4, 161-170.

Mills, E., \& Mathew, P. (2009). Monitoring-based commissioning: Benchmarking analysis of $24 \mathrm{UC} / \mathrm{CSU} / \mathrm{IOU}$ projects. Report Prepared for: California Energy Commission Public Interest Energy Research (PIER) Technology Demonstration Program. Lawrence Berkeley National Laboratory. Paper LBNL-1972E.

Mills, E., Bourassa, N., Piette, M. A., Friedman, H., Haasl, T., Powell, T., et al. (2005). The cost-effectiveness of commissioning new and existing commercial buildings: Lessons from 224 buildings. Proceedings of the National Conference on Building Commissioning. Lawrence Berkeley National Laboratory Report No. 56637. http://eetd. lbl.gov/emills/PUBS/PDF/NCBC_Mills_6Apr05.pdf. Accessed January 2010.

Motegi, N., \& Piette, M. A. (2003). Web-based energy information systems for large commercial buildings. Lawrence Berkeley National Laboratory. Paper LBNL49977. http://repositories.cdlib.org/lbnl/LBNL-49977. Accessed November 2009.

Motegi, N., Piette, M. A., Kinney, S., \& Dewey, J. (2003). Case studies of energy information systems and related technology: operational practices, costs, and benefits. Proceedings of the International Conference for Enhanced Building. http://txspace.tamu.edu/handle/1969.1/5195. Accessed September 2009.

New Buildings Institute. (2008). Energy performance of LEED for new construction buildings. Prepared for the US Green Building Council. http://www.newbuildings. org/sites/default/files/Energy_Performance_of_LEEDNC_Buildings-Final_3-4-08b.pdf. Accessed January $201 \overline{0}$. 
New Buildings Institute. (2009). Advanced metering and energy information systems. Prepared for the US Environmental Protection Agency, Grant 83378201. http://www. newbuildings.org/sites/default/files/metering-EPA\% 2083378201.pdf. Accessed January 2010.

New Buildings Institute, with the California Institute for Energy and Environment. (2009). Measured Performance Case Study: Classroom and Office Building, UC Merced. http:// www.newbuildings.org/sites/default/files/Case_Study_UCMCOB.pdf. Accessed January 2010.

Petersen, J., Shunturov, V., Janda, K., Platt, G., \& Weinberger, K. (2007). Dormitory residents reduce electricity consumption when exposed to real-time visual feedback and incentives. International Journal of Sustainability in Higher Education, 8(1), 16-33.
Piette, M. A., Kinney, S., \& Friedman, H. (2001a). EMCS and time-series energy data analysis in a large government office building. Lawrence Berkeley National Laboratory. Paper LBNL-47699. http://www.osti.gov/bridge/servlets/purl/ 787118-Qg4Jfr/native/787118.pdf. Accessed January 2010.

Piette, M. A., Kinney, S., \& Haves, P. (2001b). Analysis of an information monitoring and diagnostic system to improve building operations. Energy and Buildings, 33(8), 783791.

Webster, T. (2005). Trends in Energy Management Technologies Part 5: Effectiveness of Energy Management Systems: What the experts say and case studies reveal. Lawrence Berkeley National Laboratory. Paper LBNL-57772. http://repositories.cdlib.org/lbn1/LBNL-57772. Accessed November 2009. 